\title{
Voluntary wheel running augments aortic L-arginine transport and endothelial function in rats with chronic kidney disease
}

Christopher R. Martens

James M. Kuczmarski

Jahyun Kim

M. Brennan Harris

College of William and Mary

\section{Recommended Citation}

Martens, C. R., Kuczmarski, J. M., Kim, J., Guers, J. J., Brennan Harris, M., Lennon-Edwards, S., \& Edwards, D. G. (2014). Voluntary wheel running augments aortic l-arginine transport and endothelial function in rats with chronic kidney disease. American Journal of Physiology-Renal Physiology, 307(4), F418-F426.

This Article is brought to you for free and open access by the Arts and Sciences at W\&M ScholarWorks. It has been accepted for inclusion in Arts \& Sciences Articles by an authorized administrator of W\&M ScholarWorks. For more information, please contact scholarworks@wm.edu. 


\title{
Voluntary wheel running augments aortic L-arginine transport and endothelial function in rats with chronic kidney disease
}

\author{
Christopher R. Martens, ${ }^{1}$ James M. Kuczmarski, ${ }^{1,3}$ Jahyun Kim, ${ }^{1}$ John J. Guers, ${ }^{1}$ M. Brennan Harris, ${ }^{4}$ \\ Shannon Lennon-Edwards, ${ }^{1,2}$ and David G. Edwards ${ }^{1,3}$ \\ ${ }^{1}$ Department of Kinesiology and Applied Physiology, University of Delaware, Newark, Delaware; ${ }^{2}$ Department of Behavioral \\ Health and Nutrition, University of Delaware, Newark, Delaware; ${ }^{3}$ Department of Biological Sciences, University of \\ Delaware, Newark, Delaware; and ${ }^{4}$ Department of Kinesiology and Health Sciences, The College of William and Mary, \\ Williamsburg, Virginia
}

Submitted 8 January 2014; accepted in final form 19 June 2014

\begin{abstract}
Martens CR, Kuczmarski JM, Kim J, Guers JJ, Harris MB, Lennon-Edwards S, Edwards DG. Voluntary wheel running augments aortic L-arginine transport and endothelial function in rats with chronic kidney disease. Am J Physiol Renal Physiol 307: F418-F426, 2014. First published June 25, 2014; doi:10.1152/ajprenal.00014.2014.—Reduced nitric oxide (NO) synthesis contributes to risk for cardiovascular disease in chronic kidney disease (CKD). Vascular uptake of the NO precursor L-arginine (ARG) is attenuated in rodents with CKD, resulting in reduced substrate availability for $\mathrm{NO}$ synthesis and impaired vascular function. We tested the effect of $4 \mathrm{wk}$ of voluntary wheel running (RUN) and/or ARG supplementation on endotheliumdependent relaxation (EDR) in rats with CKD. Twelve-week-old male Sprague-Dawley rats underwent $5 / 6$ ablation infarction surgery to induce CKD, or SHAM surgery as a control. Beginning 4 wk following surgery, CKD animals either remained sedentary (SED) or received one of the following interventions: supplemental ARG, RUN, or combined RUN+ARG. Animals were euthanized 8 wk after surgery, and EDR was assessed. EDR was significantly impaired in SED vs. SHAM animals after $8 \mathrm{wk}$, in response to ACh $\left(10^{-9}-10^{-5}\right.$ $\mathrm{M})$ as indicated by a reduced area under the curve (AUC; $44.56 \pm$ 9.01 vs $100 \pm 4.58, P<0.05)$ and reduced maximal response $\left(\mathrm{E}_{\max }\right.$; $59.9 \pm 9.67$ vs. $94.31 \pm 1.27 \%, P<0.05)$. AUC was not improved by ARG treatment but was significantly improved above SED animals in both RUN and RUN+ARG-treated animals. Maximal relaxation was elevated above SED in RUN + ARG animals only. L- $\left[{ }^{3} \mathrm{H}\right]$ arginine uptake was impaired in both SED and ARG animals and was improved in RUN and RUN+ARG animals. The results suggest that voluntary wheel running is an effective therapy to improve vascular function in CKD and may be more beneficial when combined with L-arginine.
\end{abstract}

endothelial dysfunction; chronic kidney disease; L-arginine; exercise

ENDOTHELIAL DYSFUNCTION CONTRIBUTES to the development of cardiovascular disease (CVD) in patients with chronic kidney disease (CKD) and is primarily associated with a decrease in nitric oxide (NO) production and impaired endothelium-dependent relaxation (EDR) (32). The decline in endothelial function precedes the development of atherosclerosis $(17,48)$ and has been extensively studied as a potential therapeutic target to treat CVD; however, the specific mechanisms of endothelial dysfunction in CKD have not been fully elucidated. Patients with $\mathrm{CKD}$ are more likely to die of CVD than progress to

Address for reprint requests and other correspondence: D. G. Edwards, Dept. of Kinesiology and Applied Physiology, Univ. of Delaware, Newark, DE 19716 (e-mail: dge@udel.edu). end-stage renal disease $(26,42)$; therefore, novel treatments to improve endothelial function in $\mathrm{CKD}$ are needed to reduce CVD-related mortality in CKD.

Insufficient availability of the NO precursor L-arginine likely contributes to reduced NO synthesis in CKD (6). Interestingly, the use of L-arginine in studies of endothelial dysfunction in late-stage CKD has produced mixed results $(7,16)$ unlike other conditions where it has been largely effective $(8,13,15,24)$. Evidence from cell culture studies suggests that urea and other uremic toxins inhibit L-arginine uptake into endothelial cells $(52,54)$ by acting on the L-arginine transporter cationic amino acid transporter 1 (CAT-1). We have recently shown that EDR was not improved in vitro by an exogenous dose of L-arginine in an animal model of moderate to severe CKD (33). This finding was associated with reduced CAT-1 protein expression and L-arginine transport in isolated aortic rings (33) and may provide a unique explanation for why treatment with L-arginine has been ineffective in CKD. Therapies aimed at improving the utilization of L-arginine would be particularly beneficial for the restoration of endothelial function in CKD.

The endothelium is sensitive to mechanical stimuli such as shear stress that occurs with increases in blood flow. Elevated shear stress has been shown to augment L-arginine uptake in cultured endothelial cells in a dose-dependent manner (38), while exercise training has been shown to augment L-arginine uptake in the forearm vasculature of heart failure patients (36). Additionally, exercise training is well known for its ability to improve NO synthesis though activation and increased protein expression of endothelial nitric oxide synthase (eNOS), resulting in improved endothelial function $(4,23,50,53)$. The known cardiovascular benefits of aerobic exercise combined with its potential to improve the L-arginine transport system make exercise training an attractive therapy to reduce cardiovascular risk in CKD. Voluntary wheel running has previously been shown to prevent the development of vascular dysfunction in $5 / 6$ nephrectomized rats when initiated immediately after surgery (46); however, little is known about the benefits of aerobic exercise on vascular function once CKD has already been established. Similarly, the effect of exercise on the L-arginine transport system in CKD has not been tested. The purpose of this study was to determine whether $4 \mathrm{wk}$ of voluntary wheel running could improve L-arginine transport and vascular function in CKD. We hypothesized that voluntary wheel running would result in an increase in CAT-1 protein expression that would ultimately lead to an improvement in the utilization of dietary L-arginine and vascular function. 


\section{MATERIALS AND METHODS}

Ethical approval. All procedures and experiments were approved by the University of Delaware Institutional Animal Care and Use Committee and were conducted in accordance with the Public Health Service Policy regarding the Humane Care and Use of Laboratory Animals. Animals were individually housed and kept on a 12:12-h light-dark cycle and were fed a standard rat chow diet with free access to food and water.

Animal model and study groups. Twelve-week-old male SpragueDawley rats (Harlan Sprague Dawley, Indianapolis, IN) were used for this study. All rats were placed in a standard rat cage that was equipped with a running wheel for an initial period of 1 wk to allow for familiarization with the wheels. After this initial period, rats were randomly assigned to one of two groups and underwent either $5 / 6$ ablation/infarction surgery to induce CKD or a similar sham surgery to serve as a control. Animals were anesthetized using isoflurane $(1.5-5.0 \%)$ and kept on a heating pad to control body temperature during surgery. Surgery was performed using sterile technique and consisted of ligating two-thirds of the blood supply to the left kidney, followed by complete removal of the right kidney. The sham animals had both kidneys exposed and manipulated without any ablation or infarction and remained sedentary throughout the duration of the study. After 4 wk of disease development, CKD animals either remained sedentary (SED) or received one of the following interventions: supplemental L-arginine monohydrochloride (ARG; $1.25 \mathrm{~g} / \mathrm{l}$ in drinking water; Sigma-Aldrich, St. Louis, MO); voluntary wheel running (RUN); or combined RUN+ARG. The dose of L-arginine was chosen based on previous studies in which $1.25 \mathrm{~g} / \mathrm{l} \mathrm{L}$-arginine administered in the drinking water prevented the development of renal and endothelial dysfunction, respectively $(3,55)$.

Assessment of renal function. Animals were kept for 8 wk after surgery and were monitored for the development of kidney disease. This time frame is sufficient for animals to develop moderate to severe kidney disease (11), endothelial dysfunction, glomerulosclerosis, and decreased cardiac function $(30,33)$, confirming the development of both CKD and CVD in this model. Renal function was assessed in all groups by measuring urine protein excretion, serum creatinine, and blood urea nitrogen (BUN). Urine samples were obtained from an overnight collection $(16 \mathrm{~h})$ in a metabolic cage at baseline, 4 and $8 \mathrm{wk}$ postsurgery. During this time, animals were allowed access to water ad libitum; however, they were restricted from food consumption to prevent contamination of urine samples. Urine volumes were recorded, and samples were aliquotted and stored at $-80^{\circ} \mathrm{C}$ until later assay of protein concentration using the Bradford method (9). Protein excretion was calculated as the product of urine flow $(\mathrm{ml} / \mathrm{h})$ and protein concentration $(\mathrm{mg} / \mathrm{ml})$ and expressed as milligrams protein per 24 hours per 100 grams body mass.

Blood samples were obtained from the vena cava immediately before death. Samples were stored at $4{ }^{\circ} \mathrm{C}$ for $10 \mathrm{~min}$, centrifuged at $3,000 \mathrm{rpm}$ for $10 \mathrm{~min}$ at $4^{\circ} \mathrm{C}$, and serum was obtained and stored at $-80^{\circ} \mathrm{C}$ for later assay of serum creatinine and BUN. Before analysis, serum samples were filtered with $10-\mathrm{kDa}$ Amicon-Ultra centrifugal filters (EMD Millipore; Billerica, MA) to remove debris, and the filtrate was used for analysis. Plasma and urine creatinine were determined using an enzymatic creatinine assay (80340, Rat Creatinine Assay Kit, Chrystal Chem) and were used in conjunction with the urine flow rate to calculate creatinine clearance. BUN was determined from the conversion of urea to L-glutamate by urease and glutamate dehydrogenase, where a change in absorbance at $340 \mathrm{~nm}$ is directly related to BUN concentration.

Renal pathology. Kidney damage was determined from fixed kidney sections by assessing glomerulosclerosis, tubulointerstitial damage, and renal vascular damage as described in detail elsewhere (1, $35)$. Upon death, the left kidney was dissected and a transverse section was prepared and fixed in $10 \%$ formalin. Samples were sent to the Comparative Pathology Laboratory at the University of Delaware for analysis. Fixed tissue was paraffin embedded, prepared into 4- $\mu \mathrm{m}$ sections, and stained with periodic acid Schiff (PAS; 395B-1KT, Sigma-Aldrich). Staining was confirmed using positive control tissue (kidney) from another source. All tissues were microscopically evaluated by a single veterinary anatomic pathologist in a blinded fashion.

The glomerulosclerosis index (GSI) was determined at $\times 400$ magnification. Glomeruli were scored on a scale $(0-4)$ with $0=$ healthy glomeruli, $1=<25 \%$ damage, $2=25-50 \%$ damage, $3=$ $51-74 \%$ damage, and $4=>75 \%$ damage. GSI was calculated using the following equation: [(number of 1$)+2$ (number of 2$)+3$ (number of 3$)+4$ (number of 4 )/total number of glomeruli observed].

Tubulointerstitial and vascular damage scores were assessed at $\times 100$ magnification in 10 randomly selected fields/sample using the same scoring system described by Adamczak et al. (1). Tubulointerstitial damage was scored as follows: $0=$ no change; $1=$ lesions involving $<25 \%$ of area; $2=$ lesions between 25 and $50 \%$ of area; $3=$ lesions involving $>50 \%$ of area; and $4=$ lesions involving entire area. Vascular damage was scored as follows: $0=$ no wall thickening; $1=$ mild wall thickening; $2=$ moderate wall thickening; $3=$ severe wall thickening; and $4=$ fibrinoid vascular necrosis.

Preparation of vascular tissue. Animals were anesthetized with an intraperitoneal injection of ketamine/xylazine $(100 \mathrm{mg} / \mathrm{kg})$ and subsequently euthanized by exsanguination via removal of the heart. The thoracic aorta was quickly dissected and placed in ice-cold physiological salt solution (PSS; $\mathrm{NaCl}, 118.99 \mathrm{mmol}$; KCl, $4.69 \mathrm{mmol}$; $\mathrm{CaCl}_{2}-2 \mathrm{H}_{2} \mathrm{O}, 2.50 \mathrm{mmol} ; \mathrm{MgSO}_{4}-7 \mathrm{H}_{2} \mathrm{O}, 1.17 \mathrm{mmol} ; \mathrm{KH}_{2} \mathrm{PO}_{4}, 1.18$ mmol; EDTA, 0.03 mmol; glucose, 1.091 g/l; $\mathrm{NaHCO}_{3} 2.100 \mathrm{~g} / \mathrm{l} ; \mathrm{pH}$ 7.4). Aortas were cleaned of any fat and connective tissue and cut into 3-mm ring sections for assessment of vascular function or L-arginine transport as described below. The remaining aortic tissue was snapfrozen in liquid nitrogen and stored at $-80^{\circ} \mathrm{C}$ for later determination of protein expression. To determine whether wheel running resulted in improved skeletal muscle oxidative capacity, the right soleus muscle was carefully dissected, snap-frozen in liquid nitrogen, and stored at $-80^{\circ} \mathrm{C}$ for later analysis of citrate synthase activity (Sigma-Aldrich).

Vascular function studies. Vascular function was assessed in vitro using isometric ring experiments in aortic ring segments. Rings from each animal were mounted onto wire force transducers within individual organ chambers (DMT 610M, Danish Myotechnology). Rings were oxygenated with carbogen gas $\left(5 \% \mathrm{CO}_{2}-95 \% \mathrm{O}_{2}\right)$ and kept under physiological conditions at $37^{\circ} \mathrm{C}$ and $\mathrm{pH} 7.4$ in normal PSS. Vessels were stretched to a resting tension of $20 \mathrm{mN}$ and allowed to equilibrate over the course of an hour. Following equilibration, rings were constricted with a single dose of PE $\left(3 \times 10^{-7} \mathrm{M}\right)$ and relaxed with a single dose of $\mathrm{ACh}$ to test the viability of the endothelium.

Once viability was confirmed, rings were again constricted with a single dose of PE $\left(3 \times 10^{-7} \mathrm{M}\right)$ and dose dependently relaxed with cumulative doses of ACh $\left(10^{-9}-10^{-5} \mathrm{M}\right)$. Upon completion of the first dose-response experiment, rings were washed with PSS every 10 $\min$ for $1 \mathrm{~h}$ and then treated with either vehicle (PSS) or $10 \mu \mathrm{M}$ of the NOS inhibitor $N^{\omega}$-L-arginine methyl ester (L-NAME; Sigma-Aldrich) to determine whether impaired EDR was NO mediated. Rings were again constricted and dose dependently relaxed to ACh as previously described above. Finally, endothelium-independent relaxation to the NO donor SNP $\left(10^{-9}-10^{-5} \mathrm{M}\right)$ was dose dependently assessed in a subset of rings. The total duration of the vessel experiments was $\sim 8 \mathrm{~h}$. Preliminary pilot studies in our laboratory indicate that vessels remain viable for $\sim 12 \mathrm{~h}$ (data not shown).

L-Arginine transport. L-Arginine transport was assessed in aortic rings using an established in vitro technique $(25,33,43,44)$. Aortic rings were prepared into 3-mm-long ring sections, cut lengthwise into strips, and placed in HEPES buffer at $37^{\circ} \mathrm{C}$. Ring segments were then incubated for $60 \mathrm{~s}$ in HEPES buffer containing $1 \mathrm{mM} \mathrm{L}$-arginine and $10 \mu \mathrm{Ci} \mathrm{L}-\left[{ }^{3} \mathrm{H}\right]$ arginine. A separate set of rings was incubated in buffer containing $1 \mathrm{mM}$ L-arginine but no L- $\left[{ }^{3} \mathrm{H}\right]$ arginine to serve as a control. Rings were then washed with ice-cold PBS and homogenized in RIPA buffer. Tissue homogenates were analyzed for radioactivity 
(disintegrations per minute) by liquid scintillation counting using a Packard Tri-Carb 2900-TR liquid scintillation counter, and protein content was determined using a BCA protein assay (Thermo Scientific).

Western blotting. Protein expression of CAT-1, PKC $\alpha$, eNOS, and phosphorylated eNOS (Ser 1177) were determined by Western blotting. Aortic tissue samples were homogenized at a 1:20 dilution in lysis buffer containing $(150 \mathrm{mM} \mathrm{NaCl}, 1.0 \%$ Triton $\mathrm{X}-100,0.5 \%$ SDS, and $50 \mathrm{mM}$ Tris. $\mathrm{HCl}, \mathrm{pH}$ 8.0) with added protease and phosphatase inhibitor cocktails (Sigma-Aldrich) using glass-on-glass homogenization. Homogenates were centrifuged at $10,000 \mathrm{~g}$ for $10 \mathrm{~min}$ at $4^{\circ} \mathrm{C}$, and the supernatant was extracted and prepared for analysis. Protein concentration was determined using a Bradford assay (9), and samples were diluted in sample buffer containing $\beta$-mercaptoethanol and boiled for $5 \mathrm{~min}$. Samples were loaded into either 12 (CAT-1; $\mathrm{PKC} \alpha$ ) or $8 \%$ (eNOS; eNOS-P1177) Tris. $\mathrm{HCl}$ gels and electrophoresed for $60 \mathrm{~min}$ at $100 \mathrm{~V}$. Gels were transferred to a nitrocellulose membrane, blocked, and immunoblotted with the primary antibody for the protein of interest: CAT-1 (sc-66825, 1:200; Santa Cruz Biotechnology), PKC $\alpha$ (sc-8393, 1:100; Santa Cruz Biotechnology), eNOS (BD 610297, 1:1,000), or eNOS-P1177(BD 612393, 1:500). Membranes were washed and incubated with the appropriate recommended secondary antibody, treated with ECL developing reagent (Thermo Scientific), and developed on film. Data are presented as intensity relative to $\beta$-actin (sc-81178, 1:2,000; Santa Cruz Biotechnology). Western blots were quantified using Image $\mathbf{J}$ software from the National Institutes of Health.

Statistical analysis. Urinary protein excretion was analyzed using a two-way repeated-measures ANOVA. All other data were analyzed using a one-way ANOVA, and Tukey's post hoc test was performed to assess differences between groups. Dose-response curves were generated for all vascular function data using GraphPad Prism 5.0 software and normalized to percent relaxation. Dose-response curves were fit with a nonlinear regression line, and $\operatorname{LogEC}_{50}$, maximal relaxation or constriction $\left(\mathrm{E}_{\max }\right)$, and the area under the curve (AUC) were determined. The $\alpha$ level was set at 0.05 , and all data are presented as means $\pm \mathrm{SE}$.

\section{RESULTS}

Table 1 contains body mass data and measurements of renal function. Body mass was significantly reduced in SED and ARG-treated animals compared with SHAM and was restored by RUN and RUN+ARG interventions (Table 1). Urinary protein excretion was unaltered in SHAM animals and was progressively elevated in all of the CKD groups at 4 and $8 \mathrm{wk}$ postsurgery. Serum creatinine and BUN were significantly elevated above SHAM animals to similar levels at $8 \mathrm{wk}$ in all CKD groups. No differences in renal function were observed among any of the CKD groups at any time point, indicating no effect of treatment on renal function. Similarly, GSI and tubulointerstitial and vascular damage scores were significantly elevated above SHAM animals in all groups with CKD and were unaffected by intervention (Fig. 1). Animals in the RUN and RUN+ARG group ran a similar distance over the course of the 4-wk intervention (Table 2). Citrate synthase activity was significantly attenuated in SED and RUN+ARG animals compared with SHAM and was reduced in all other groups, but not significantly. Animals in the ARG and RUN+ARG group consumed a similar amount of L-arginine in their drinking water (Table 2).

Vascular function studies. The complete EDR dose-response to $\mathrm{ACh}$ in aortic rings is presented in Fig. $2 A$. The role of NO in mediating the EDR response to ACh was confirmed in all animals by an abolished relaxation response to the NOS inhibitor L-NAME. Some rings continued to constrict in the presence of L-NAME, likely due to ACh-mediated constriction of smooth muscle cells in the absence of endothelium-derived NO production (22). EDR to ACh was significantly impaired in both SED and ARG animals as indicated by a reduction in the AUC of the dose-response curve (Fig. 2B). Running improved AUC above SED animals in both RUN and RUN+ARGtreated animals. RUN + ARG had the greatest effect overall and was significantly improved above ARG animals (Fig. 2B). The $\mathrm{E}_{\max }$ of ACh was also significantly attenuated in both SED and ARG-treated animals. $E_{\max }$ was not significantly different from SHAM animals and was improved above SED in the RUN + ARG group only (Fig. 2C). Endothelium-independent relaxation to SNP was not impaired in SED animals compared with SHAM and was not improved by any of the treatments. A small but significant reduction in $\operatorname{LogEC}_{50}$ to SNP was observed in RUN + ARG animals (Table 3).

Tissue analysis. L-Arginine transport was attenuated in both SED and ARG-treated animals as indicated by a reduction in aortic uptake of $\mathrm{L}-\left[{ }^{3} \mathrm{H}\right]$ arginine compared with SHAM controls. L-Arginine transport was improved above SED animals in the RUN group. The greatest improvement occurred in the $\mathrm{RUN}+\mathrm{ARG}$ animals in which L-arginine transport was greater than both SED and ARG animals (Fig. 3A). Protein expression of the L-arginine transporter CAT-1 was attenuated in SED animals compared with SHAM and was unaltered by any of the treatments (Fig. 3B), despite the observation of an overall improvement in L-arginine transport. We observed a significant increase in PKC $\alpha$ protein expression in our SED animals only, whereas both RUN and RUN + ARG animals returned to similar levels as SHAM (Fig. 4). Protein expression of eNOS was not significantly different among groups; however, phosphorylation of eNOS at ser-1177 was attenuated in sedentary CKD animals compared with SHAM and was not improved by any of the treatments (Fig. 5).

Table 1. Body mass and renal function measurements

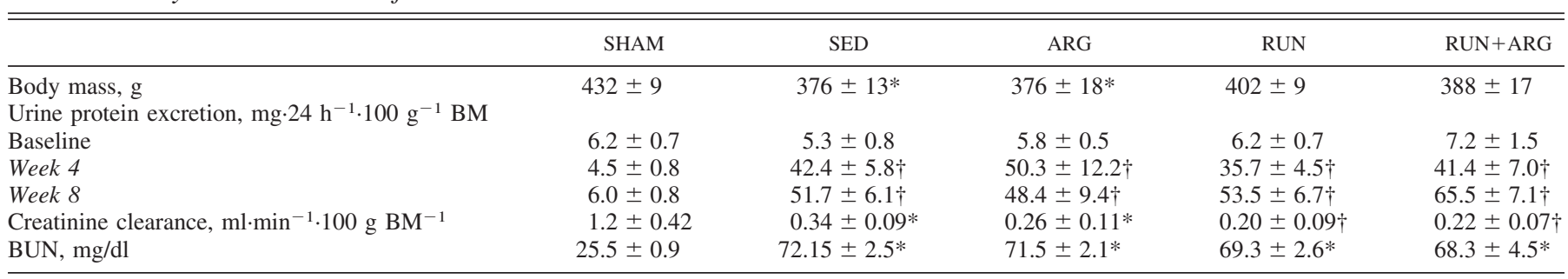

Values are mean \pm SE. BM, body mass; BUN blood urea nitrogen; SED, sedentary rats; RUN, wheel-running rats; ARG, $L$-arginine-treated rats. $* P<0.05$ vs. SHAM. $\dagger P<0.001$ vs. SHAM; $n=5-8$ /group. 

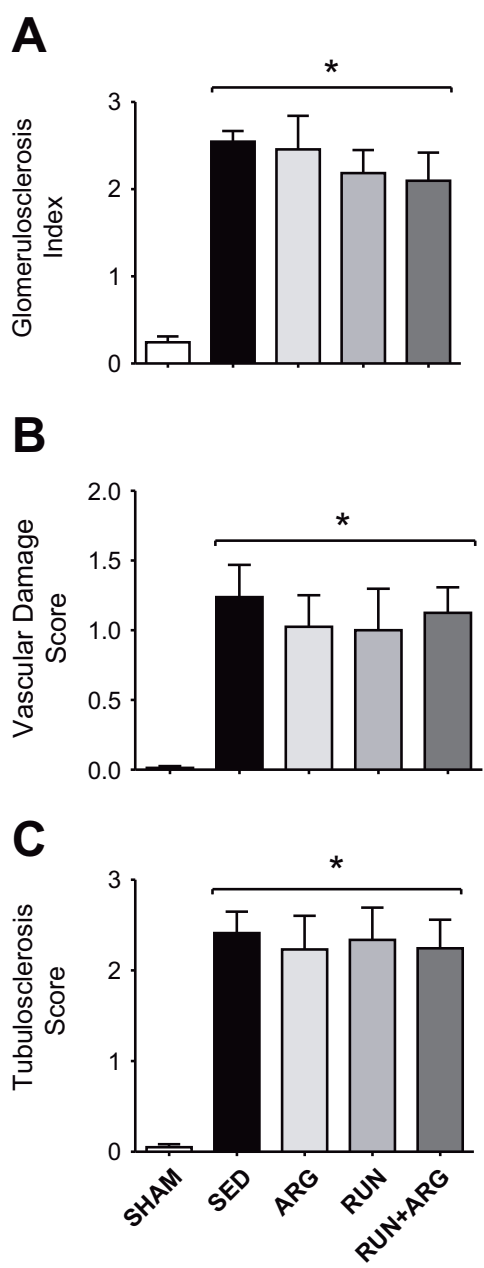

\section{DISCUSSION}

The primary finding of this study is that 4 wk of low-volume voluntary wheel running reversed vascular dysfunction in rats with CKD. This effect was greatest in animals that received L-arginine in combination with the wheel running intervention but was not seen in animals that received L-arginine alone. The improvement in vascular function was not associated with increased CAT-1 protein expression but was accompanied by augmented L-arginine transport and a reduction in $\mathrm{PKC} \alpha$ protein expression. The results of this study demonstrate that even small increases in physical activity may be a beneficial therapy to reverse vascular dysfunction in CKD and suggest that exercise may work in part by improving the vascular uptake of L-arginine.

The beneficial effect of exogenous L-arginine on vascular function has been well documented across a wide range of pathologies, including hypercholesterolemia, diabetes mellitus,
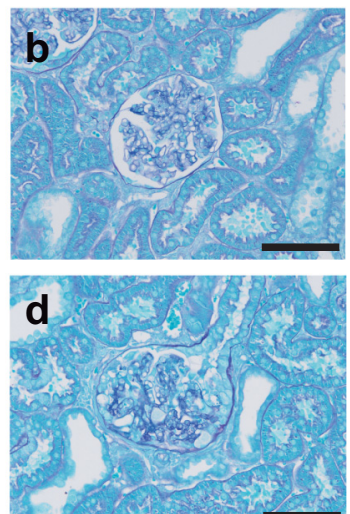

Fig. 1. The glomerulosclerosis index (GSI; $A)$, vascular damage score $(B)$, and tubulointerstitial damage score $(C)$ were elevated above SHAM in all groups with CKD and were unaffected by intervention. Representative glomeruli were classified using glomerulosclerosis scores $[0=$ normal glomerulus $(a),+1=<25 \%$ damage $(b),+2=25-50 \%$ damage $(c),+3=51-74 \%$ damage $(d)$, and $+4=75-100 \%$ damage $(e)]$ based upon the degree of mesangial and basement membrane thickening and tuft collapse. Experimental infarction results are also shown in variable degrees of interstitial fibrosis and tubular dilatation, as well as marked vascular medial hypertrophy (arrowheads; $f$ ) in cortical vessels adjacent to affected glomeruli (bottom right). ${ }^{*} P<0.05$ vs. SHAM; $n=8$ /group.

Table 2. Intervention characteristics

\begin{tabular}{|c|c|c|c|c|c|}
\hline & SHAM & SED & ARG & RUN & $\mathrm{RUN}+\mathrm{ARG}$ \\
\hline
\end{tabular}

Values are mean $\pm \mathrm{SE} * P<0.05$ vs. SHAM; $n=8$. 
A

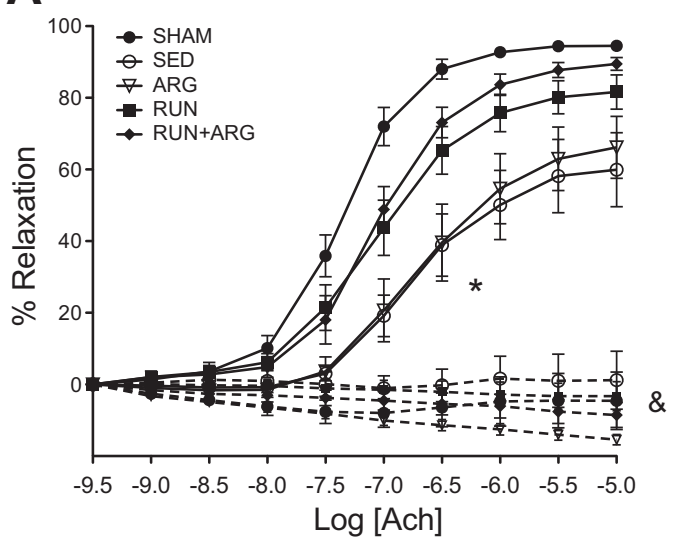

B

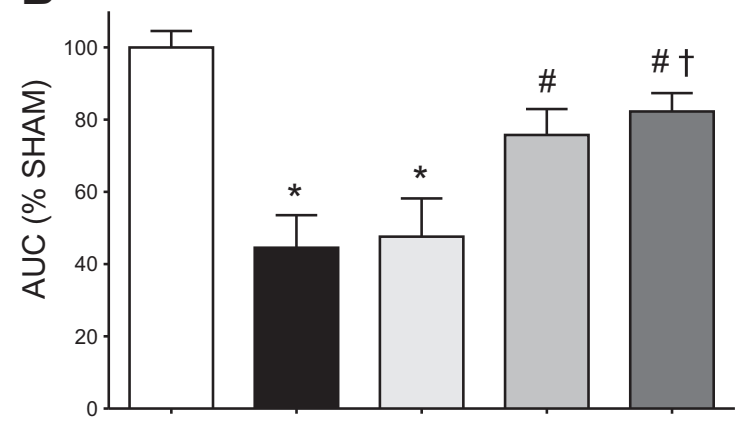

C

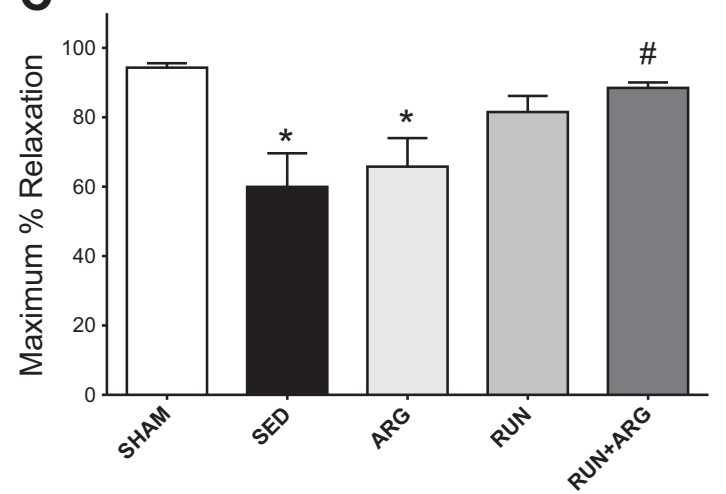

Fig. 2. A: endothelium-dependent relaxation (EDR) of aortic rings to ACh under control (solid lines) and $N^{\omega}$-L-arginine methyl ester (L-NAME)-treated (dashed lines) conditions. ${ }^{*} P<0.05$ vs. respective control $(B)$ area under the curve (AUC), and maximal EDR response to ACh $(C)$. $* P<0.05$ vs. SHAM. $\# P<0.05$ vs. sedentary (SED) rats. $\dagger P<0.05$ vs. L-arginine-treated rats (ARG); $n=8 /$ group.

important, these findings do not provide insight into the role of L-arginine in vascular function once CKD has already been established. Unfortunately, clinical studies in patients with CKD have not been as successful $(7,12,16,18)$. In the present study, we initiated L-arginine treatment $4 \mathrm{wk}$ after surgery to ensure that renal and vascular impairments had already developed. Using this model, we were unable to improve vascular dysfunction in our ARG-treated animals, suggesting that L-arginine is not effective at reversing endothelial dysfunction once CKD has already been established. Our findings are in agreement with clinical studies that demonstrate no improvement in endothelium-dependent dilation response to either an acute infusion of L-arginine in adult predialysis patients (16) or to oral supplementation with L-arginine in children with chronic renal failure (7).

The lack of improvement with L-arginine is not entirely surprising and may explain a unique mechanism by which endothelial function is impaired in CKD. The transport of extracellular L-arginine into the endothelium occurs primarily through CAT-1 via a sodium- and $\mathrm{pH}$-independent mechanism (14). L-Arginine transport has been shown to be impaired in endothelial cells cultured in uremic plasma (54) as well as in animal models of CKD $(25,33,43)$ and is accompanied by a decline in CAT-1 protein expression in the vasculature. We confirmed these findings in the present paper as demonstrated by a significant reduction in CAT-1 protein expression and $\mathrm{L}-\left[{ }^{3} \mathrm{H}\right]$ arginine uptake in SED and ARG-treated animals. In this context, impaired vascular transport of L-arginine potentially explains the lack of improvement in endothelial function observed in our ARG treatment group.

CAT-1 forms a caveolar complex with the NO-producing enzyme eNOS $(31,34)$, suggesting a mechanism by which extracellular L-arginine is delivered directly to eNOS at the endothelial cell surface. NO synthesis is improved by endothelial shear stress such as that which occurs with increased blood flow during exercise. This adaptation occurs at the onset of exercise via an increase in eNOS phosphorylation at ser-1179 (4) as well as in response to long-term exercise training through increased eNOS expression (45). Interestingly, we did not detect a significant attenuation in eNOS protein expression; however, phosphorylation at ser-1177 was decreased in all groups with CKD and was unaltered by voluntary wheel running. The duration and intensity of the wheel-running intervention may not have been great enough to cause changes in eNOS expression or activation. Improvements in vascular function with exercise, however, are not limited to changes in eNOS expression and activity, as porcine aortic endothelial cells exposed to $40 \mathrm{~min}$ of shear stress exhibited improved L-arginine uptake (38). Similarly, $8 \mathrm{wk}$ of aerobic and lightresistance exercise training augmented forearm vascular uptake of L-arginine in humans with congestive heart failure (36). Our results confirm the beneficial effects of exercise on vascular function, as animals that received access to a running wheel (RUN) improved their vascular function by $\sim 30 \%$ above SED animals. Additionally, L-arginine supplementation may have provided additional benefits when given in combination with wheel running, as animals in the RUN+ARG group increased vascular function by nearly $40 \%$ above both SED and ARGtreated animals. These results suggest that physical activity is not only capable of augmenting endothelial function in CKD

Table 3. Endothelium-independent response to SNP

\begin{tabular}{llrrr}
\hline \hline & $n$ & \multicolumn{1}{c}{ AUC } & \multicolumn{1}{c}{$E_{\max }$} & \multicolumn{1}{c}{$\operatorname{LogEC}_{50}$} \\
\hline SHAM & 7 & $100 \pm 3.68$ & $100.57 \pm 0.89$ & $-8.56 \pm 0.13$ \\
SED & 8 & $87.65 \pm 4.75$ & $97.36 \pm 1.11$ & $-8.17 \pm 0.15$ \\
ARG & 7 & $84.45 \pm 7.26$ & $99.11 \pm 1.21$ & $-8.03 \pm 0.22$ \\
RUN & 8 & $85.15 \pm 2.30$ & $98.02 \pm 0.72$ & $-8.08 \pm 0.11$ \\
RUN+ARG & 7 & $80.83 \pm 4.02$ & $96.56 \pm 1.20$ & $-7.77 \pm 0.15 *$ \\
\hline
\end{tabular}

Values are means \pm SE; $n$, number of animals; AUC, area under the curve; $\mathrm{E}_{\max }$, maximal relaxation or constriction. ${ }^{*} P<0.05$ vs. SHAM. 
A

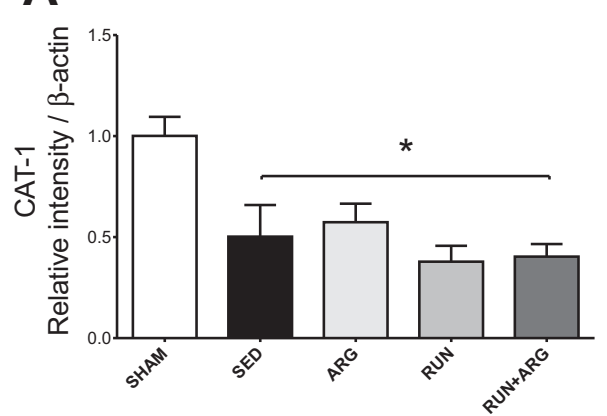

CAT-1

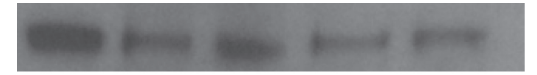

$\beta$-actin
B

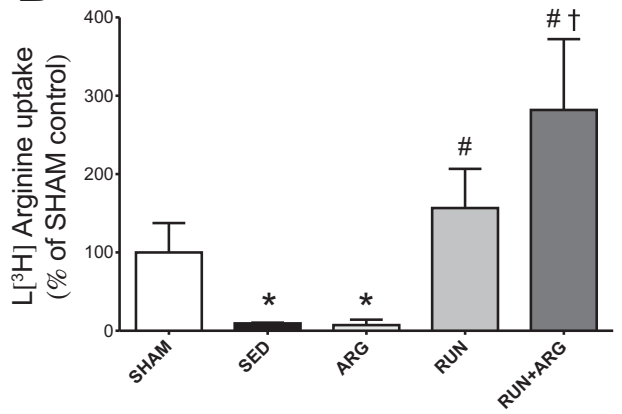

Fig. 3. A: aortic cationic amino acid transporter 1 (CAT-1) protein expression normalized to $\beta$-actin assessed by Western blotting; $n=8$ /group. $B$ : L- $\left[{ }^{3} \mathrm{H}\right]$ arginine uptake, with disintegrations/min (DPM) normalized to protein content; $n=5$ /group. $* P<0.05$ vs. SHAM. $\# P<0.05$ vs. SED. $\dagger P<0.05$ vs. ARG.

$70 \mathrm{kDa}$

$43 \mathrm{kDa}$

but that it may do so in part by improving the utilization of L-arginine.

Our initial hypothesis was that exercise would improve vascular function by increasing protein expression of the L-arginine transporter CAT-1. Interestingly, the improved relaxation observed in RUN and RUN+ARG animals did not appear to occur through an increase in CAT-1 protein expression; however, wheel running did improve CAT-1 transport activity as evidenced by an increase in $\mathrm{L}-\left[{ }^{3} \mathrm{H}\right]$ arginine uptake. This suggests that exercise mediates an increase in L-arginine uptake through a posttranslational modification of CAT-1. Previous work by Ingbir et al. (25) reported similar results in which treatment of uremic rats with rosiglitazone improved L-arginine uptake in aortic rings without increasing CAT-1 protein expression. Rosiglitazone treatment was associated with a decrease in aortic PKC $\alpha$ protein expression and an increase in CAT-1 phosphorylation, suggesting that PKC $\alpha$ is an important mediator of CAT-1 transport activity (25). In the present study, we observed an increase in $\mathrm{PKC} \alpha$ expression in
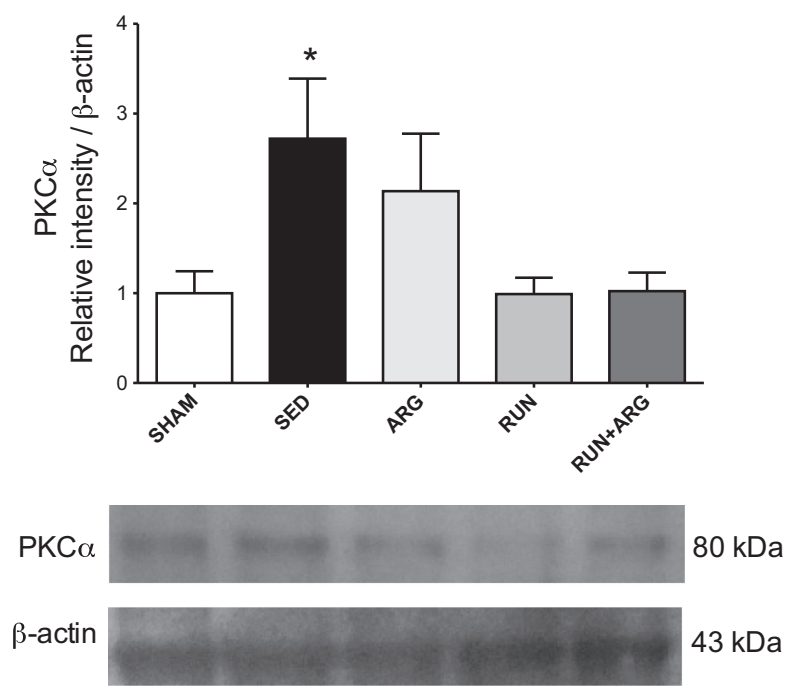

Fig. 4. PKC $\alpha$ protein expression normalized to $\beta$-actin assessed by Western blotting. $* P<0.05$ vs. SHAM; $n=8 /$ group. the aortas of SED animals whereas levels were returned to similar levels as SHAM animals in both RUN and RUN+ARG treatment groups. It should be noted that these analyses were conducted in whole aortic homogenates and that additional insight could be gained by comparing these data to vessels with denuded endothelium to confirm the localization of CAT-1/ $\mathrm{PKC} \alpha$ within the endothelium.

The exact mechanism by which $\mathrm{PKC} \alpha$ mediates a reduction in L-arginine transport is not entirely clear. Activation of PKC $\alpha$ with PMA has been shown to result in internalization of CAT-1 to the cytosol in Xenopus laevis oocytes and was shown to occur independently of CAT-1 phosphorylation (41). In another study, conducted in pulmonary artery endothelial cells, PMA activation of $\mathrm{PKC} \alpha$ reduced $\mathrm{L}$-arginine uptake without changing the expression or subcellular distribution of CAT-1 (29). Whether PKC $\alpha$ mediates a decrease in L-arginine transport through a direct reduction in CAT-1 catalytic activity or by inducing its translocation to the cytosol in CKD is not completely understood. In our experiments, the magnitude of increase in $\mathrm{L}-\left[{ }^{3} \mathrm{H}\right]$ arginine uptake with wheel running exceeded that of SHAM control animals, suggesting exercise may influence L-arginine transport at least in part through a direct alteration in CAT-1 catalytic activity; however, future work is needed to elucidate this mechanism. Furthermore, our results are only associative at this point and do not definitively link the observed improvements in L-arginine transport to the improvements in endothelial function observed with exercise. Future studies in which CAT-1 expression or transport activity is experimentally manipulated in the presence or absence of physical activity would provide valuable insight into the relative contribution of increased L-arginine transport to the overall improvement in vascular function.

In conclusion, 4 wk of low-volume voluntary wheel running improved vascular function in rats with CKD through a mechanism that is potentially related to the observed improved L-arginine transport activity. This mechanism was not related to an increase in CAT- 1 protein expression but was associated with a reduction in $\mathrm{PKC} \alpha$ protein expression, suggesting posttranslational modification of CAT-1. Treatment with exogenous L-arginine has not been successful in patients with CKD, and our data suggest that exercise may play an important role 


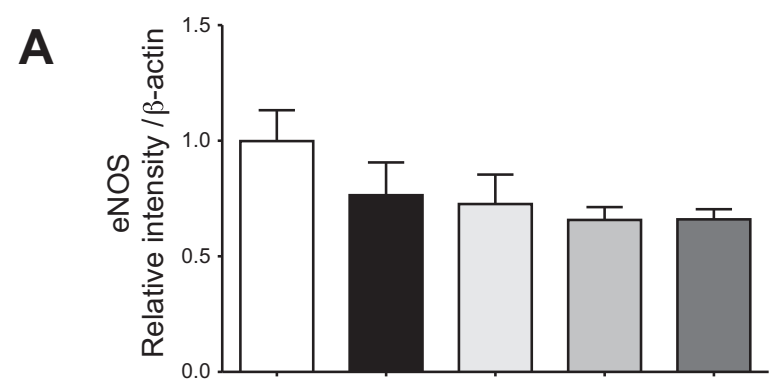

B
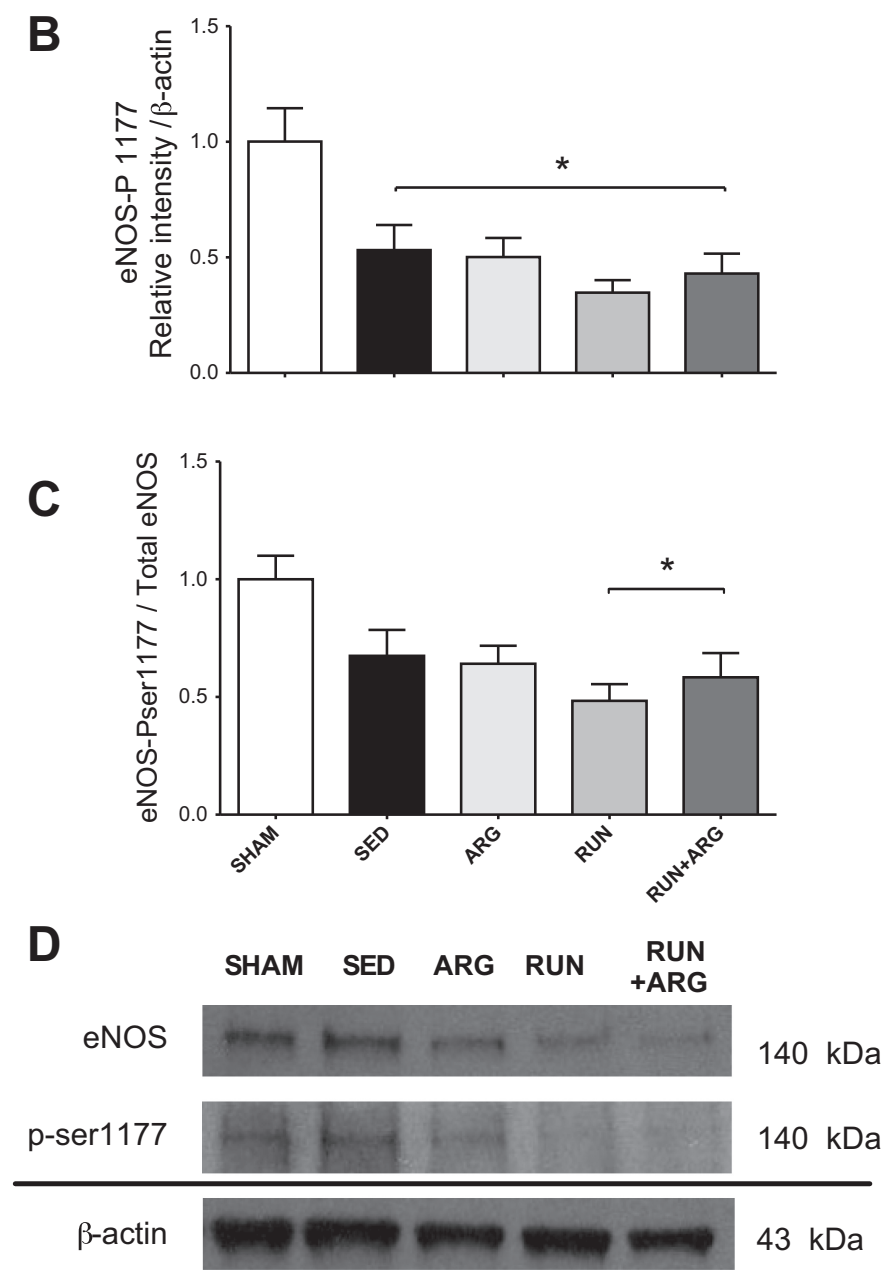

Fig. 5. Total endothelial nitric oxide synthase (eNOS; $A$ ) and phosphorylated eNOS $(\operatorname{ser}-1177 ; B)$ in aorta normalized to $\beta$-actin and abundance of phosphorylated eNOS relative to total eNOS assessed by Western blot $(C)$ with representative blots $(D) ; n=8$ /group. ${ }^{*} P<0.05$ vs. SHAM.

in the regulation of the L-arginine-NO axis. Future work should consider the L-arginine transport mechanism as a therapeutic target to treat CVD in humans with CKD and should further explore the role of increased physical activity in improving vascular health.

Translational perspectives. Our findings are particularly interesting given the low volume of physical activity recorded in both running groups and suggest that even small increases in physical activity may reverse vascular dysfunction in CKD. Adams et al. (2) reported running distances of $\sim 7 \mathrm{~km} /$ day in both SHAM rats and rats that underwent $5 / 6$ nephrectomy when animals were given access to a running wheel during the initial stages of disease development. In our study, improvements in vascular function were detected in both running groups with distances of only $\sim 9 \mathrm{~km}$ over the course of the entire 4-wk intervention period. This finding has important clinical relevance, as patients with CKD typically have poor tolerance to exercise accompanied by a sedentary lifestyle (28). The ability to achieve robust improvements in cardiovascular function with only minor increases in daily levels of physical activity could have a profound effect on the management of CVD in patients with CKD. Studies in previously sedentary older adults with baseline vascular dysfunction have demonstrated improvements in NO-mediated dilation after only 3 mo of low-intensity aerobic exercise (i.e., brisk walking) (19), providing evidence that the endothelium can respond to lowintensity training. Our data suggest that patients with CKD may benefit from similar low-intensity physical activity.

In addition to a decline in vascular function, skeletal muscle wasting also occurs in patients with CKD, resulting in a decrease in body mass. In contrast to the general population, a higher body mass index is associated with reduced CVD risk in patients with CKD (5). In the present study, we observed a decrease in body mass in both groups of CKD animals that remained sedentary; however, body mass was not decreased in animals that received access to a running wheel. This finding suggests that a low volume of physical activity is capable of protecting against the CKD-associated decline in body mass and may provide added protection against cardiovascular events.

Despite the many health benefits of regular physical activity, exercise is not typically prescribed in patients with CKD (27, 28, 49). Furthermore, guidelines for the prescription of exercise in CKD are less established (27). The absence of clinical exercise prescription may stem from concern over the safety of exercise training in patients with CKD (21); however, our data suggest that low-volume physical activity improves vascular function in the absence of changes in renal function or structural damage. Overall, our findings in a preclinical model of CKD have the potential to lead to clinically relevant studies regarding the use of exercise as an adjunct therapy to reduce the burden of CVD in patients with CKD.

\section{ACKNOWLEDGMENTS}

The authors acknowledge and thank Dr. Erin Brannick and Joanne Kramer of the CANR Comparative Pathology Laboratory at the University of Delaware for assistance with renal pathology analyses.

\section{GRANTS}

This work was supported by National Institutes of Health Grant P20 RR016472-11 (D. G. Edwards) and a 2012 ACSM Foundation Grant (C. R. Martens).

\section{DISCLOSURES}

No conflicts of interest, financial or otherwise, are declared by the authors.

\section{AUTHOR CONTRIBUTIONS}

Author contributions: C.R.M., M.B.H., S.L.-E., and D.G.E. provided conception and design of research; C.R.M., J.M.K., J.K., and J.J.G. performed experiments; C.R.M. analyzed data; C.R.M. and D.G.E. interpreted results of experiments; C.R.M. prepared figures; C.R.M. drafted manuscript; C.R.M., J.M.K., M.B.H., S.L.-E., and D.G.E. edited and revised manuscript; C.R.M., J.M.K., J.K., J.J.G., M.B.H., S.L.-E., and D.G.E. approved final version of manuscript. 


\section{REFERENCES}

1. Adamczak M, Gross ML, Krtil J, Koch A, Tyralla K, Amann K, Ritz E. Reversal of glomerulosclerosis after high-dose enalapril treatment in subtotally nephrectomized rats. $J$ Am Soc Nephrol 14: 2833-2842, 2003.

2. Adams G, Zhan C, Haddad F, Vaziri N. Voluntary exercise during chronic renal failure in rats. Med Sci Sports Exerc 37: 557-562, 2005.

3. Ashab I, Peer G, Blum M, Wollman Y, Chernihovsky T, Hassner A, Schwartz D, Cabili S, Silverberg D, Iaina A. Oral administration of L-arginine and captopril in rats prevents chronic renal failure by nitric oxide production. Kidney Int 47: 1515-1521, 1995.

4. Balligand J, Feron O, Dessy C. eNOS activation by physical forces: from short-term regulation of contraction to chronic remodeling of cardiovascular tissues. Physiol Rev 89: 481-534, 2009.

5. Bansal N, Vittinghoff E, Plantinga L, Hsu C. Does chronic kidney disease modify the association between body mass index and cardiovascular disease risk factors? J Nephrol 25: 317-324, 2012.

6. Baylis C. Nitric oxide deficiency in chronic kidney disease. Am J Physiol Renal Physiol 294: F1-F9, 2008.

7. Bennett-Richards K, Kattenhorn M, Donald A, Oakley G, Varghese Z, Bruckdorfer K, Deanfield J, Rees L. Oral L-arginine does not improve endothelial dysfunction in children with chronic renal failure. Kidney Int 62: 1372-1378, 2002.

8. Bode-Boger SM, Muke J, Surdacki A, Brabant G, Boger RH, Frolich JC. Oral L-arginine improves endothelial function in healthy individuals older than 70 years. Vasc Med 8: 77-81, 2003.

9. Bradford M. Rapid and sensitive method for quantitation of microgram quantities of protein utilizing principle of protein-dye binding. Anal Biochem 72: 248-254, 1976.

10. Chauhan A, More R, Mullins P, Taylor G, Petch M, Schofield P. Aging-associated endothelial dysfunction in humans is reversed by L-arginine. J Am Coll Cardiol 28: 1796-1804, 1996.

11. Chen G, Baylis C. In vivo renal arginine release is impaired throughout development of chronic kidney disease. Am J Physiol Renal Physiol 298: F95-F102, 2010

12. Cherla G, Jaimes E. Role of L-arginine in the pathogenesis and treatment of renal disease. J Nutr 134: 2801S-2806S, 2004.

13. Clarkson P, Adams MR, Powe AJ, Donald AE, McCredie R, Robinson J, McCarthy SN, Keech A, Celermajer DS, Deanfield JE. Oral L-arginine improves endothelium-dependent dilation in hypercholesterolemic young adults. J Clin Invest 97: 1989-1994, 1996.

14. Closs EI, Boissel J, Habermeier A, Rotmann A. Structure and function of cationic amino acid transporters (CATs). J Membr Biol 213: 67-77, 2006.

15. Cooke JP, Andon NA, Girerd XJ, Hirsch AT, Creager MA. Arginine restores cholinergic relaxation of hypercholesterolemic rabbit thoracic aorta. Circulation 83: 1057-1062, 1991.

16. Cross JM, Donald AE, Kharbanda R, Deanfield JE, Woolfson RG, MacAllister RJ. Acute administration of L-arginine does not improve arterial endothelial function in chronic renal failure. Kidney Int 60: 2318-2323, 2001

17. Davignon J, Ganz P. Role of endothelial dysfunction in atherosclerosis. Circulation 109: 27-32, 2004

18. De Nicola L, Bellizzi V, Minutolo R, Andreucci M, Capuano A, Garibotto G, Corso G, Andreucci V, Cianciaruso B. Randomized, double-blind, placebo-controlled study of arginine supplementation in chronic renal failure. Kidney Int 56: 674-684, 1999.

19. DeSouza C, Shapiro L, Clevenger C, Dinenno F, Monahan K, Tanaka H, Seals D. Regular aerobic exercise prevents and restores age-related declines in endothelium-dependent vasodilation in healthy men. Circulation 102: 1351-1357, 2000.

20. Feng Q, Lu X, Fortin A, Pettersson A, Hedner T, Kline R, Arnold J. Elevation of an endogenous inhibitor of nitric oxide synthesis in experimental congestive heart failure. Cardiovasc Res 37: 667-675, 1998.

21. Fuiano G, Mancuso D, Cianfrone P, Comi N, Mazza G, Marino F, Fuiano L, Zamboli P, Caglioti A, Andreucci M. Can young adult patients with proteinuric $\operatorname{IgA}$ nephropathy perform physical exercise? Am J Kidney Dis 44: 257-263, 2004

22. Furchgott RF, Zawadzki JV. The obligatory role of endothelial-cells in the relaxation of arterial smooth-muscle by acetylcholine. Nature 288 : 373-376, 1980.

23. Harris MB, Mitchell BM, Sood SG, Webb RC, Venema RC. Increased nitric oxide synthase activity and Hsp90 association in skeletal muscle following chronic exercise. Eur J Appl Physiol 104: 795-802, 2008.
24. Holowatz LA, Thompson CS, Kenney WL. L-arginine supplementation or arginase inhibition augments reflex cutaneous vasodilatation in aged human skin. J Physiol 574: 573-581, 2006.

25. Ingbir M, Schwartz IF, Shtabsky A, Filip I, Reshef R, Chernichovski T, Levin-Iaina N, Rozovski U, Levo Y, Schwartz D. Rosiglitazone improves aortic arginine transport, through inhibition of PKC $\alpha$, in uremic rats. Am J Physiol Renal Physiol 295: F471-F477, 2008.

26. Isbel NM, Haluska B, Johnson DW, Beller E, Hawley C, Marwick TH. Increased targeting of cardiovascular risk factors in patients with chronic kidney disease does not improve atheroma burden or cardiovascular function. Am Heart J 151: 745-753, 2006

27. Johansen K. Exercise and chronic kidney disease-current recommendations. Sports Med 35: 485-499, 2005.

28. Johansen KL, Painter P. Exercise in individuals with CKD. Am J Kidney Dis 59: 123-134, 2012.

29. Krotova K, Zharikov S, Block E. Classical isoforms of PKC as regulators of CAT-1 transporter activity in pulmonary artery endothelial cells. Am J Physiol Lung Cell Mol Physiol 284: L1037-L1044, 2003.

30. Kuczmarski JM, Martens CR, Lennon-Edwards SL, Edwards DG. Cardiac function and tolerance to ischemia-reperfusion injury in chronic kidney disease. Nephrol Dial Transplant [Epub ahead of print].

31. Li C, Huang W, Harris M, Goolsby J, Venema R. Interaction of the endothelial nitric oxide synthase with the CAT-1 arginine transporter enhances NO release by a mechanism not involving arginine transport. Biochem J 386: 567-574, 2005.

32. London GM, Pannier B, Agharazii M, Guerin AP, Verbeke FHM, Marchais SJ. Forearm reactive hyperemia and mortality in end-stage renal disease. Kidney Int 65: 700-704, 2004.

33. Martens CR, Kuczmarski JM, Lennon-Edwards S, Edwards DG. Impaired L-arginine uptake but not arginase contributes to endothelial dysfunction in rats with chronic kidney disease. $J$ Cardiovasc Pharmacol 63: $40-48,2014$

34. McDonald K, Zharikov S, Block E, Kilberg M. A caveolar complex between the cationic amino acid transporter 1 and endothelial nitric-oxide synthase may explain the "arginine paradox". J Biol Chem 272: 31213 31216, 1997.

35. Moningka NC, Sindler AL, Muller-Delp JM, Baylis C. Twelve weeks of treadmill exercise does not alter age-dependent chronic kidney disease in the Fisher 344 male rat. $J$ Physiol 589: 6129-6138, 2011.

36. Parnell M, Holst D, Kaye D. Augmentation of endothelial function following exercise training is associated with increased L-arginine transport in human heart failure. Clin Sci 109: 523-530, 2005.

37. Pieper G, Peltier B. Amelioration by L-arginine of a dysfunctional arginine nitric-oxide pathway in diabetic endothelium. J Cardiovasc Pharmacol 25: 397-403, 1995.

38. Posch K, Schmidt K, Graier W. Selective stimulation of L-arginine uptake contributes to shear stress-induced formation of nitric oxide. Life Sci 64: 663-670, 1999.

39. Reyes A, Purkerson M, Karl I, Klahr S. Dietary supplementation with L-arginine ameliorates the progression of renal-disease in rats with subtotal nephrectomy. Am J Kidney Dis 20: 168-176, 1992.

40. Reyes AA, Karl IE, Klahr S. Role of arginine in health and in renal disease. Am J Physiol Renal Fluid Electrolyte Physiol 267: F331-F346, 1994.

41. Rotmann A, Stand D, Martine U, Closs E. Protein kinase C activation promotes the internalization of the human cationic amino acid transporter hCAT-1-a new regulatory mechanism for hCAT-1 activity. J Biol Chem 279: 54185-54192, 2004.

42. Sarnak MJ, Levey AS, Schoolwerth AC, Coresh J, Culleton B, Hamm LL, McCullough PA, Kasiske BL, Kelepouris E, Klag MJ, Parfrey P, Pfeffer M, Raij L, Spinosa DJ, Wilson PW; American Heart Association Councils on Kidney in Cardiovascular Disease, High Blood Pressure Research, Clinical Cardiology, and Epidemiology and Prevention. Kidney disease as a risk factor for development of cardiovascular disease-a statement from the American Heart Association Councils on Kidney in Cardiovascular Disease, High Blood Pressure Research, Clinical Cardiology, and Epidemiology and Prevention. Circulation 108: 2154-2169, 2003.

43. Schwartz I, Ayalon R, Chernichovski T, Reshef R, Chernin G, Weinstein T, Litvak A, Levo Y, Schwartz D. Arginine uptake is attenuated through modulation of cationic amino-acid transporter-1, in uremic rats. Kidney Int 69: 298-303, 2006. 
44. Schwartz IF, Chernichovski T, Schwartz D. Aortic arginine transport is attenuated, through post-translational modulation of CAT-1 by PKC alpha, in old male rats. Vasc Med 15: 55-59, 2010.

45. Sessa WC, Pritchard K, Seyedi N, Wang J, Hintze TH. Chronic exercise in dogs increases coronary vascular nitric-oxide production and endothelial cell nitric oxide synthase gene expression. Circ Res 74: 349-353, 1994.

46. Shelkovnikov S, Summers SM, Elahimehr R, Adams G, Purdy RE, Vaziri ND. Effect of exercise training on aortic tone in chronic renal insufficiency. Am J Hypertens 21: 564-569, 2008.

47. Shin S, Mohan S, Fung H. Intracellular L-arginine concentration does not determine NO production in endothelial cells: Implications on the "Larginine paradox". Biochem Biophys Res Commun 414: 660-663, 2011.

48. Sitia S, Tomasoni L, Atzeni F, Ambrosio G, Cordiano C, Catapano A, Tramontana S, Perticone F, Naccarato P, Camici P, Picano E, Cortigiani L, Bevilacqua M, Milazzo L, Cusi D, Barlassina C, Sarzi-Puttini P, Turiel M. From endothelial dysfunction to atherosclerosis. Autoimmun Rev 9: 830-834, 2010.

49. Stride M. Exercise and the patient with chronic kidney disease. Br J Hosp Med 72: 200-204, 2011.
50. Tanabe T, Maeda S, Miyauchi T, Iemitsu M, Takanashi M, Irukayama-Tomobe Y, Yokota T, Ohmori H, Matsuda M. Exercise training improves ageing-induced decrease in eNOS expression of the aorta. Acta Physiol Scand 178: 3-10, 2003.

51. Tousoulis D, Davies G, Tentolouris C, Crake T, Toutouzas P. Coronary stenosis dilatation induced by L-arginine. Lancet 349: 1812-1813, 1997.

52. Wagner L, Klein JD, Sands JM, Baylis C. Urea transporters are distributed in endothelial cells and mediate inhibition of L-arginine transport. Am J Physiol Renal Physiol 283: F578-F582, 2002.

53. Walther C, Gielen S, Hambrecht R. The effect of exercise training on endothelial function in cardiovascular disease in humans. Exerc Sport Sci Rev 32: 129-134, 2004.

54. Xiao S, Wagner L, Mahaney J, Baylis C. Uremic levels of urea inhibit L-arginine transport in cultured endothelial cells. Am J Physiol Renal Physiol 280: F989-F995, 2001.

55. Yamamizu K, Shinozaki K, Ayajiki K, Gemba M, Okamura T. Oral administration of both tetrahydrobiopterin and L-arginine prevents endothelial dysfunction in rats with chronic renal failure. J Cardiovasc Pharmacol 49: 131-139, 2007.

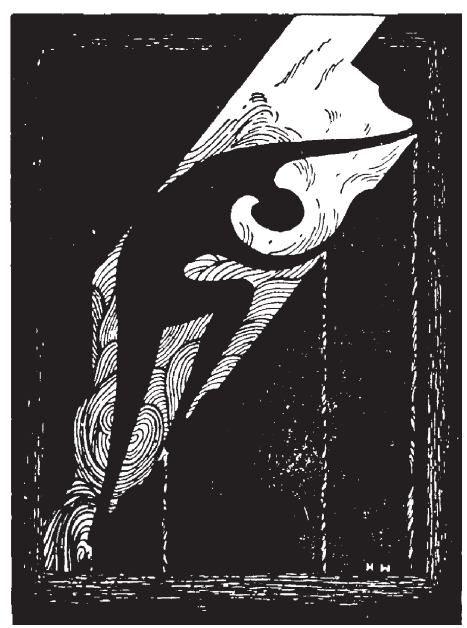

